\title{
Article
}

\section{Accurate Determination of Ion Polarizabilities in Aqueous Solutions}

Minglun Li, Bilin Zhuang, Yuyuan Lu, Zhen-Gang Wang, and Lijia An

J. Phys. Chem. B, Just Accepted Manuscript • Publication Date (Web): 08 Jun 2017

Downloaded from http://pubs.acs.org on June 12, 2017

\section{Just Accepted}

"Just Accepted" manuscripts have been peer-reviewed and accepted for publication. They are posted online prior to technical editing, formatting for publication and author proofing. The American Chemical Society provides "Just Accepted" as a free service to the research community to expedite the dissemination of scientific material as soon as possible after acceptance. "Just Accepted" manuscripts appear in full in PDF format accompanied by an HTML abstract. "Just Accepted" manuscripts have been fully peer reviewed, but should not be considered the official version of record. They are accessible to all readers and citable by the Digital Object Identifier (DOI®). "Just Accepted" is an optional service offered to authors. Therefore, the "Just Accepted" Web site may not include all articles that will be published in the journal. After a manuscript is technically edited and formatted, it will be removed from the "Just Accepted" Web site and published as an ASAP article. Note that technical editing may introduce minor changes to the manuscript text and/or graphics which could affect content, and all legal disclaimers and ethical guidelines that apply to the journal pertain. ACS cannot be held responsible for errors or consequences arising from the use of information contained in these "Just Accepted" manuscripts. 


\title{
Accurate Determination of Ion Polarizabilities in Aqueous Solutions
}

\author{
Minglun Li, ${ }^{\dagger}, \ddagger$ Bilin Zhuang, ${ }^{\top}$ Yuyuan Lu, ${ }^{*} \dagger$ Zhen-Gang Wang, ${ }^{*}$ I and Lijia An ${ }^{\dagger}$ \\ $\dagger$ State Key Laboratory of Polymer Physics and Chemistry, Changchun Institute of Applied \\ Chemistry, Chinese Academy of Sciences, Changchun 130022, People's Republic of China \\ $\ddagger$ University of Chinese Academy of Sciences, Beijing 100049, People’s Republic of China \\ 【Division of Chemistry and Chemical Engineering, California Institute of Technology, \\ Pasadena, CA 91125, United States \\ E-mail: yylu@ciac.ac.cn; zgw@caltech.edu
}

Phone: +86-0431-85262138; +1-626-395-4647. Fax: +86-0431-85262126; +1-626-568-8743 


\begin{abstract}
We present a novel method for obtaining salt polarizabilities in aqueous solutions based on our recent theory for the refractive index of salt solutions, which predicts a linear relationship between the refractive index and the salt concentration at low concentrations, with a slope determined by the intrinsic values of the salt polarizability and the density of the solution. Here we apply this theory to determine the polarizabilities of 32 strong electrolyte salts in aqueous solutions from refractive index and density measurements. Setting $\mathrm{Li}^{+}$as the standard ion, we then determine the polarizabilities of seven cations $\left(\mathrm{Na}^{+}, \mathrm{K}^{+}, \mathrm{Rb}^{+}, \mathrm{Cs}^{+}, \mathrm{Ca}^{2+}, \mathrm{Ba}^{2+}\right.$ and $\left.\mathrm{Sr}^{2+}\right)$ and seven anions $\left(\mathrm{F}^{-}\right.$, $\mathrm{Cl}^{-}, \mathrm{Br}^{-}, \mathrm{I}^{-}, \mathrm{ClO}_{4}^{-}, \mathrm{NO}_{3}^{-}$and $\mathrm{SO}_{4}^{-}$), which can be used as important reference data. We investigate the effect of temperature on salt polarizabilities, which decreases slightly with increasing temperature. The ion polarizability is found to be proportional to the cube of bare ionic radius $\left(r_{\text {bare }}^{3}\right)$ for univalent ions, but the relationship does not hold for multivalent ions. Contrary to findings of Krishnamurti, we find no significant linear relationship between ion polarizability and the square of the atomic number $\left(N^{2}\right)$ for smaller ions.
\end{abstract}

\title{
I. INTRODUCTION
}

Ion polarizability in aqueous solution, defined as the ratio of the induced dipole moment of an ion to the local electric field, ${ }^{1}$ is of fundamental importance in physical chemistry, ${ }^{2}$ atmospheric science ${ }^{3,4}$ and biophysics. ${ }^{5,6}$ Ion polarizability has been invoked as a key factor in the explanation of the Hofmeister series - the systematic trend of different ions with the same valency in their ability to precipitate proteins or macromolecules from aqueous solutions. ${ }^{5-9}$ Ion polarizability also plays an important role in interfacial phenomena involving aqueous ionic solutions. For example, it affects the interfacial adsorption of ions, ${ }^{4,10,11}$ and thus the interfacial tension. ${ }^{12,13}$ at water/air or water/oil interfaces. It is also known to influence the charge transfer involving ions adsorbed to the water/vapor interfaces ${ }^{14}$ and the interfacial 
viscosity of ionic solutions. ${ }^{4,5}$ Clearly, accurate and reliable values for the ion polarizability are critical to the understanding and modeling of these systems.

Using quantum mechanics, Pauling systematically calculated the polarizabilities of simple ions in the gaseous phase, ${ }^{15}$ and these estimates have been widely used for various calculations involving ion polarizabilities. ${ }^{16}$ However, ion polarizabilities in the aqueous phase can be significantly different from their corresponding the gas-phase values ${ }^{17,18}$ due to the strong interactions between the charge of ions and the permanent or induced dipoles of solvent molecules. ${ }^{19}$ Unfortunately, Pauling's theoretical framework cannot be readily extended to calculate polarizabilities of ions to account for these interactions in the aqueous phase.

Due to the difficulties in predicting aqueous-phase ion polarizabilities with analytical theory, computational simulation has become an important tool for predicting ion polarizabilities in aqueous solutions. A range of methods with different levels of complexity have been developed, including density functional theory with Car-Parrinello molecular dynamics, ${ }^{18,20}$ and ab initio quantum calculations with Car-Parrinello molecular dynamics. ${ }^{21}$ However, computer simulations necessarily involve approximations in the modeling and treatment of the ions and the solvent species. For example, Masia found that the calculated value of the chloride ion polarizability has non-negligible dependence on the system size. ${ }^{20}$ To obtain the polarizability of the sulfate dianion, Jungwirth et al. ${ }^{21,22}$ had to treat the $\mathrm{SO}_{4}^{2-}\left(\mathrm{H}_{2} \mathrm{O}\right)_{4}$ cluster as a special species, where the four coordinating water molecules are modeled differently from the bulk water. Their reported value of the polarizability of the sulfate dianion, $7 \AA^{3}$, which is considerably larger than our measured value of $4.418 \AA^{3}$, may possibly be due to such approximations.

As a result of these challenges, obtaining ion polarizabilities via experimental measurements is desirable. Ion polarizability in solution is related to the molar refractivity $R:^{23}$

$$
R=\frac{4 \pi}{3} N_{\mathrm{A}} \alpha_{\text {salt }}
$$


where $N_{\mathrm{A}}$ is Avogadro's constant and $\alpha_{\text {salt }}$ denotes the sum of polarizabilities of the solvated ions in cgs units. For aqueous salt solutions, $R$ can be related to the refractive index and volumes of the liquid before and after addition of salt, according to the Clausius-Mossotti equation ${ }^{24-26}$

$$
R=\frac{1}{c_{\mathrm{salt}}}\left(\frac{n^{2}-1}{n^{2}+2}-\frac{n_{\mathrm{water}}^{2}-1}{n_{\mathrm{water}}^{2}+2} \frac{V_{\mathrm{water}}}{V_{\mathrm{sol}}}\right) .
$$

Here, $c_{\text {salt }}$ is the molar salt concentration, with unit mol $/ \mathrm{L} ; V_{\text {water }}$ and $n_{\text {water }}$ are the volume and refractive index of pure water, respectively, before addition of salt; and $V_{\text {sol }}$ and $n$ are, respectively, the volume and refractive index of the aqueous mixture solution after addition of salt. The refractivity measured from using Eq. (2) at finite concentrations is generally concentration dependent, reflecting the interaction and interference between the ions in the solution. To obtain the intrinsic values of the salt polarizability at infinite dilution, one needs to extrapolate the measured $R$ from finite concentration to $c_{\text {salt }}=0$. Then the intrinsic salt polarizability is obtained from Eq. (1). We shall refer to this approach as the direct extrapolation (DE) method. ${ }^{19,27}$ Such a method, though valid in principle, suffers from two sources of error. First, in the experimentally relevant ranges of salt concentration, the relation between salt polarizability and salt concentration is usually not simply linear. And second, at extreme dilution, the error bars in the molar refractivity can become significantly large (see Figure S1 for more details).

Recently, we proposed a simple theoretical formula describing the concentration dependence of refractive indices of liquid mixtures containing salt ions. ${ }^{28}$ One of the key results in that work is the prediction of the slope of the refractive index with respect to salt concentration at low concentrations, in terms of the polarizability of the ionic species and the infinite dilution value of the partial molar volume of the salt, which is determined by extrapolation of the solution volume to zero salt concentration by including the Debye-Hückel correction. ${ }^{29,30}$ Our predicted slopes for several different salts and different compositions of the water-acetonitrile liquid mixtures are in excellent agreement with experimental data. Since both the refractive index and the volume data can be easily and accurately measured 
with modern instrumentation, ${ }^{16,31-34}$ which allows easy and accurate determination of the limiting slope, we can use the theory in reverse to obtain the polarizability of the salts from measured values of the limiting slope. In the current work, we employ this strategy to obtain the polarizabilities of 32 strong electrolyte salts in aqueous solution and compare our results with earlier results obtained through other methods. By setting $\mathrm{Li}^{+}$as the standard ion, we determine the value of the polarizability for seven common cations and seven common anions $-\mathrm{Na}^{+}, \mathrm{K}^{+}, \mathrm{Rb}^{+}, \mathrm{Cs}^{+}, \mathrm{Ca}^{2+}, \mathrm{Ba}^{2+}$ and $\mathrm{Sr}^{2+} ; \mathrm{F}^{-}, \mathrm{Cl}^{-}, \mathrm{Br}^{-}, \mathrm{I}^{-}, \mathrm{ClO}_{4}^{-}, \mathrm{NO}_{3}^{-}$and $\mathrm{SO}_{4}^{-}$.

In addition, we analyze the effects of temperature, ionic radius and atomic number on ion polarizabilities. While Heydweiller ${ }^{25}$ conducted some preliminary studies on the effect of temperature on salt polarizabilities, we perform a more systematic examination at six different temperatures from $25^{\circ} \mathrm{C}$ to $65^{\circ} \mathrm{C}$. Iwadate et al. ${ }^{31}$ found that the polarizabilities of ions in univalent molten salts are proportional to the cube of the bare ionic radius $\left(r_{\text {bare }}^{3}\right)$, which is in accord with theoretical predictions of Machmer. ${ }^{35}$ However, for multivalent ions, theoretical data by Pauling ${ }^{15}$ and Tessman et al. ${ }^{34}$ showed that this correlation does not hold. Thus far, there has been no study on the relation between ion polarizability and $r_{\text {bare }}^{3}$ in aqueous ions. Finally, Krishnamurti ${ }^{36,37}$ proposed a linear correlation between the square of the atomic number and ion polarizabilities in aqueous solutions, but the mechanism of this relationship remains unclear. The extensive data set we have obtained allows us to address these issues.

The rest of this paper is organized as follows. In Section II, we present the key results from our previous work, ${ }^{28}$ in particular, the working formula for relating the salt polarizability to the limiting slope of the refractive index with respect to salt concentration. In Section III, we describe the materials and experimental methods. In Section IV, we present our results for salt and ion polarizabilities and discuss the effects of temperature, ionic radius, and atomic number. We provide some concluding remarks in Section V. 


\section{THEORETICAL BACKGROUND}

In our recent paper ${ }^{28}$ we derived a linear relationship between the refractive index and the salt concentration of an electrolyte at low concentrations in water-acetonitrile-salt systems. Here, we briefly review the main analysis, as applied to the simpler salt-water mixtures. We start with a rearranged form of Eq. (2) using the polarizability of the salt:

$$
\frac{n^{2}-1}{n^{2}+2}=\frac{4 \pi}{3} \rho_{\text {salt }} \alpha_{\text {salt }}+\frac{\rho_{\text {water }}}{\rho_{\text {water }}^{(0)}} \frac{n_{\text {water }}^{2}-1}{n_{\text {water }}^{2}+2}
$$

In this equation, $\rho_{\text {water }}^{(0)}$ is the number density of water molecules in pure water, and $\rho_{\text {water }}$ and $\rho_{\text {salt }}$ are the number density of water and salt in the solution, respectively. We use $\alpha_{\text {salt }}$ to denote the stoichiometric sum of the polarizabilities of the solvated ions. For example, for a dissociated ionic salt of formula $\mathrm{C}_{m} \mathrm{~A}_{n}, \alpha_{\text {salt }}=m \alpha_{+}+n \alpha_{-}$, where $\alpha_{+}$and $\alpha_{-}$are polarizabilities of the cation and the anion, respectively.

In order to conveniently relate the formula to experimental measurements, we introduce the mole fraction of the salt, $x_{\text {salt }}=\rho_{\text {salt }} /\left(\rho_{\text {salt }}+\rho_{\text {water }}\right)$, as the concentration variable. In addition, because the volume is more directly measurable than the number density, it is convenient to write the number density of the species to the measured volume data. Hence, we introduce two volume quantities for the salt-water mixture: the molar volume of water $\left(V_{\text {water }}^{(0)}=N_{\mathrm{A}} / \rho_{\text {water }}^{(0)}\right)$ and the volume of the salt-water mixture when salt is added to 1 mole of water $\left(V_{\text {sol }}\right)$. The number densities of water $\left(\rho_{\text {water }}\right)$ and salt $\left(\rho_{\text {salt }}\right)$ in the mixture are related to $V_{\text {sol }}$ through

$$
\rho_{\mathrm{water}}=\frac{N_{\mathrm{A}}}{V_{\mathrm{sol}}}
$$

and

$$
\rho_{\text {salt }}=\frac{x_{\text {salt }}}{1-x_{\text {salt }}} \frac{N_{\mathrm{A}}}{V_{\text {sol }}}
$$


Rewriting Eq. (3) in terms of volume quantities and mole fraction of the salt, we obtain

$$
\frac{n^{2}-1}{n^{2}+2}=\frac{V_{\mathrm{water}}^{(0)}}{V_{\mathrm{sol}}}\left(\frac{x_{\text {salt }}}{1-x_{\text {salt }}} \beta \alpha_{\text {salt }}+\frac{n_{\text {water }}^{2}-1}{n_{\text {water }}^{2}+2}\right)
$$

where $\beta=\left(4 \pi N_{\mathrm{A}}\right) /\left(3 V_{\text {water }}^{(0)}\right)$.

The volume $V_{\text {sol }}$ differs from $V_{\text {water }}^{(0)}$ due to the addition of salt. At low salt concentrations, we may write

$$
V_{\text {sol }}=V_{\text {water }}^{(0)}\left(1+\gamma^{\prime} x_{\text {salt }}+\eta x_{\text {salt }}^{\frac{3}{2}}\right) .
$$

where it can be easily seen that $\gamma^{\prime} V_{\text {water }}^{(0)}$ corresponds to the infinite dilution limit of the partial molar volume of the salt, which is defined as $\bar{V}_{\text {salt }} \equiv \lim _{x_{\text {salt }} \rightarrow 0}\left[\left(V_{\text {sol }}-V_{\text {water }}^{(0)}\right) / x_{\text {salt }}\right]$. The $x_{\text {salt }}^{3 / 2}$ term is due to ion-ion interactions according to the Debye-Hückel limiting law. ${ }^{29,30}$ In theory, the constant $\eta$ only depends on the solvent and the valency of the ions, but experimentally, it was shown that different salts yield different values of $\eta \cdot{ }^{30}$ So we treat $\eta$ as a fitting parameter.

For univalent salts such as $\mathrm{NaCl}, \mathrm{KCl}$, and $\mathrm{KBr}$, the mixture volume exhibits very good linearity with the salt concentration at low salt concentrations $\left(x_{\text {salt }}<0.04\right)$. Therefore, in our previous work, ${ }^{28}$ we neglected the nonlinear dependence of mixture volume on salt concentration and modeled the volume of the salt-water mixture using the following simpler relationship

$$
V_{\text {sol }}=V_{\text {water }}^{(0)}\left(1+\gamma x_{\text {salt }}\right) .
$$

However, for some salts such as $\mathrm{NaF}$ and $\mathrm{MgSO}_{4}$, the concentration range accessible experimentally may not be sufficiently low for the simple linear relationship to be applicable. In such situations, the $x_{\text {salt }}^{3 / 2}$ term cannot be neglected, and the value of $\gamma$ determined experimentally by using Eq. (8) can be significantly different from $\gamma^{\prime}$. The Debye-Hückel limiting law is fitted for all the salts we study in this work,${ }^{30}$ and for better accuracy, we obtain $\gamma^{\prime}$ by fitting our volume data to Eq. (7) for all salts.

Substituting Eq. (7) into Eq. (6) and expanding the resulting expression to the first order 
in $x_{\text {salt }}$, the following simplified expression for low salt concentrations is obtained

$$
n=n_{\text {water }}+K x_{\text {salt }}
$$

with the slope $K$ given by

$$
K=\frac{n_{\text {water }}^{2}+2}{6 n_{\text {water }}}\left[\left(n_{\text {water }}^{2}+2\right) \beta \alpha_{\text {salt }}-\left(n_{\text {water }}^{2}-1\right) \gamma^{\prime}\right] .
$$

Thus, knowing $\gamma^{\prime}$ from fitting the volume data, and $K$ from fitting the refractive index data, we can determine the value of the salt polarizability. Since $\gamma^{\prime}$ and $K$ can be obtained quite readily and accurately from the experimental data, Eq. (10) provides an important method for accurate determination of salt polarizabilities in aqueous solutions. The relevant data from our experimental measurements are listed in Table 1 and discussed in Section IV.

\section{EXPERIMENTS}

\section{Chemicals.}

Deionized and doubly distilled water was used in the experiments. Except for salts with crystalloid water, hexahydrate strontium chloride $\left(\mathrm{SrCl}_{2} \cdot 6 \mathrm{H}_{2} \mathrm{O}\right.$, Vetec, $99.0 \%$, reagent grade), hexahydrate magnesium nitrate $\left(\mathrm{MgNO}_{3} \cdot 6 \mathrm{H}_{2} \mathrm{O}\right.$, Aladdin, $99 \%$, $\left.\mathrm{AR}\right)$ and dihydrate barium chloride $\left(\mathrm{BaCl}_{2} \cdot 2 \mathrm{H}_{2} \mathrm{O}\right.$, Aladdin, $99.99 \%$, metal basis), the other salts were dried in a vacuum oven $\left(80^{\circ} \mathrm{C}, 133 \mathrm{~Pa}\right)$ for 2 hours prior to use. These salts included sodium fluoride (NaF, Aladdin, 99.99\%, metal basis), potassium fluoride (KF, Aladdin, 99.5\%, GR), cesium fluoride (CsF, Aladdin, 99\%, AR), lithium chloride ( $\mathrm{LiCl}$, Vetec, reagent grade), sodium chloride ( $\mathrm{NaCl}$, Vetec, 99.0\%, reagent grade), potassium chloride ( $\mathrm{KCl}$, Vetec, 99.0\%, reagent grade), rubidium chloride ( $\mathrm{RbCl}$, Aladdin, 99.5\%, AR), cesium chloride $(\mathrm{CsCl}, \mathrm{Al}-$ addin, $99 \%$, AR), magnesium chloride $\left(\mathrm{MgCl}_{2}\right.$, Aldrch, powder $\left.\leq 70 \mu \mathrm{m}\right)$, calcium chloride $\left(\mathrm{CaCl}_{2}\right.$, Vetec, $96 \%$, reagent grade), lithium bromide ( $\mathrm{LiBr}$, Vetec, reagent grade), sodi- 
um bromide (NaBr, Vetec, 98.0\%, reagent grade), potassium bromide (KBr, Vetec, 99.0\%, reagent grade), cesium bromide ( $\mathrm{CsBr}$, Aladdin, 99.5\%, metal basis), magnesium bromide $\left(\mathrm{MgBr}_{2}\right.$, Aldrich, 98\%), sodium iodide (NaI, Aladdin, 99.5\%, AR), potassium iodide (KI, Aladdin, 99.5\%, GR), cesium iodide (CsI, Aladdin, 99.9\%, metal basis), lithium nitrate $\left(\mathrm{LiNO}_{3}\right.$, Aladdin, 99.9\%, metal basis), sodium nitrate $\left(\mathrm{NaNO}_{3}\right.$, Sigma-Aldrch, 99.0\%, ACS), potassium nitrate $\left(\mathrm{KNO}_{3}\right.$, Sigma-Aldrch, 99.0\%, ACS), lithium perchlorate $\left(\mathrm{LiClO}_{4}\right.$, Aladdin, 98\%, AR), sodium perchlorate $\left(\mathrm{NaClO}_{4}\right.$, Aladdin, 98\%, ACS), barium perchlorate $\left(\mathrm{Ba}\left(\mathrm{ClO}_{4}\right)_{2}\right.$, Aladdin, 97\%, anhydrous), lithium sulfate $\left(\mathrm{Li}_{2} \mathrm{SO}_{4}\right.$, Aladdin, $97 \%$, anhydrous, titration), sodium sulfate $\left(\mathrm{Na}_{2} \mathrm{SO}_{4}\right.$, Vetec, $99.0 \%$, reagent grade), potassium sulfate $\left(\mathrm{K}_{2} \mathrm{SO}_{4}\right.$, Vetec, 99.0\%, reagent grade), cesium sulfate $\left(\mathrm{Cs}_{2} \mathrm{SO}_{4}\right.$, Aladdin, 99.9\%, metal basis) and magnesium sulfate $\left(\mathrm{MgSO}_{4}\right.$, Sigma-Aldrch, 99.5\%, anhydrous). All mixtures were prepared by weighing the masses, with an uncertainty of $\pm 0.0001 \mathrm{~g}$ due to evaporation.

\section{Measurements.}

The sample was kept in Thermo HAAKE SC150-A5B circulating water bath (Newington, U. S. A.) with a digital temperature control unit to maintain the required temperature within $\pm 0.1^{\circ} \mathrm{C}$ at experimental temperature $\left(25^{\circ} \mathrm{C}, 35^{\circ} \mathrm{C}, 37^{\circ} \mathrm{C}, 45^{\circ} \mathrm{C}, 55^{\circ} \mathrm{C}\right.$, and $\left.65^{\circ} \mathrm{C}\right)$ for at least one hour to reach thermostatic equilibrium before being taken out for density and refractive index measurement.

The volumes of the solutions were calculated by the density data with known mass of solutions (mass divided by the density). The densities of the solutions were measured to a precision of $0.0001 \mathrm{~g} / \mathrm{cm}^{3}$ using an Anton Paar DMA 4100M digital temperature-control densitometer (Graz, Austria). The densitometer was calibrated periodically with doubledistilled water at the experimental temperature. The densitometer has self-temperature control function (Thermo Balance $\left.{ }^{T M}\right)$ that sets the required temperature $\left(25^{\circ} \mathrm{C}, 35^{\circ} \mathrm{C}, 37^{\circ} \mathrm{C}\right.$, $45^{\circ} \mathrm{C}, 55^{\circ} \mathrm{C}$, and $65^{\circ} \mathrm{C}$ ) within $\pm 0.01^{\circ} \mathrm{C}$. The refractive indices of the solutions were measured at experimental temperature ambient pressure using an Insmark IR120 plus (Shanghai, P. 


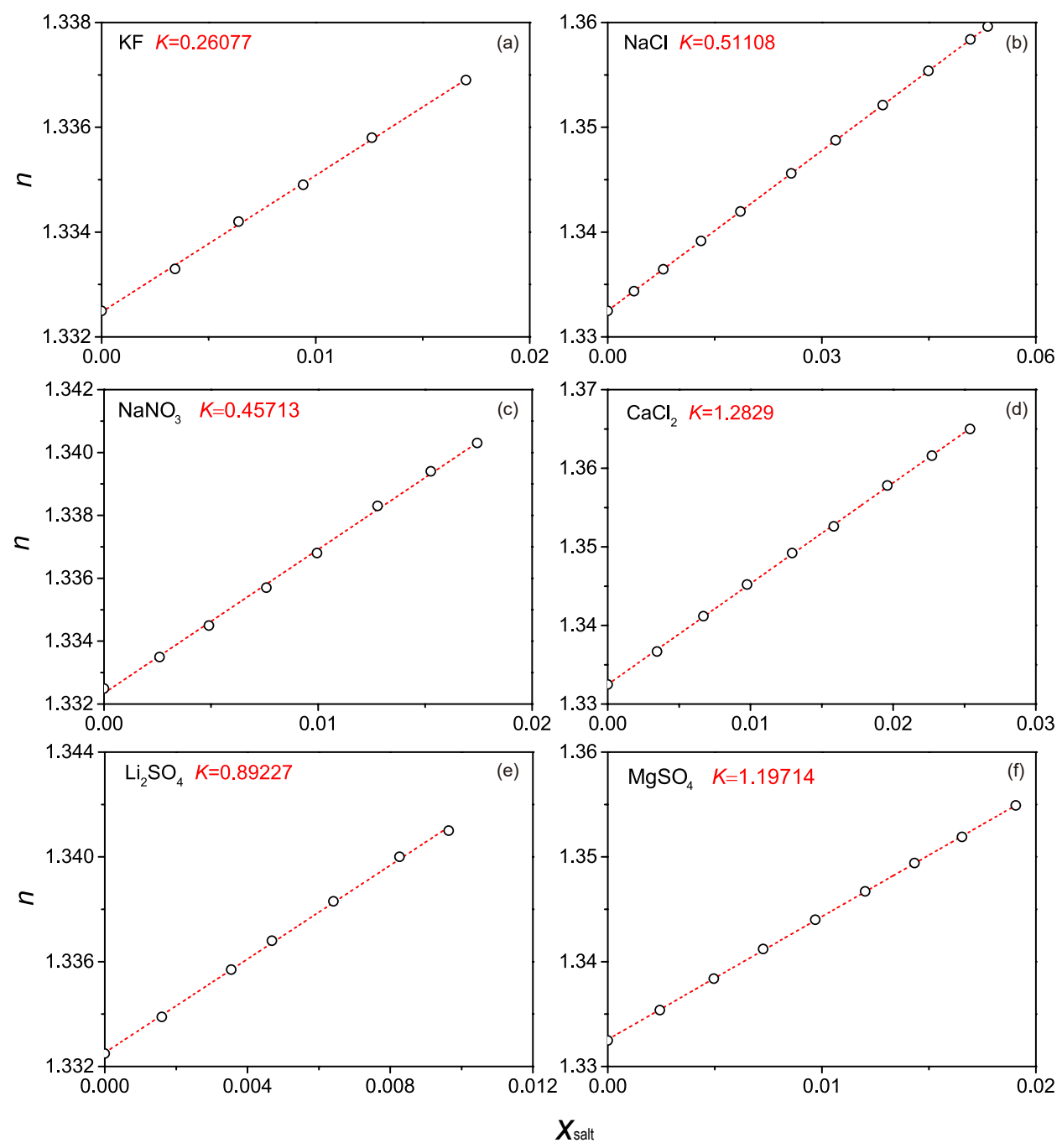

Figure 1: Refractive index $n$ vs. salt mole fraction $x_{\text {salt }}$ for (a) KF, (b) $\mathrm{NaCl}$ (c) $\mathrm{NaNO}_{3}$, (d) $\mathrm{CaCl}_{2}$, (e) $\mathrm{Li}_{2} \mathrm{SO}_{4}$ and (f) $\mathrm{MgSO}_{4}$ at $25^{\circ} \mathrm{C}$. The slopes $(K)$ from linear fitting of the data are also included.

R. C. ) thermostatic digital refractometer $\left( \pm 0.1^{\circ} \mathrm{C}\right)$ at the wavelength of the D-line of sodium $(589.3 \mathrm{~nm})$. The precision of the measurement is estimated to be \pm 0.0001 . The density and refractive index data reported are the average of three separate samples. All measurements were made at ambient pressure.

For a given salt solution at a given temperature, the following protocol was used: First, we plotted the refractive indices $n v s$. the salt mole fraction $x_{\text {salt }}$ in the dilute concentration range and obtained the slope $K$ according to Eqs. (10) and (9) as shown in Figure 1. Next, we plotted $V_{\text {sol }} / V_{\text {water }}^{(0)}$ vs. $x_{\text {salt }}$ in the same concentration range to to obtain $\gamma^{\prime}$ according to 
Eqs. (7) (see Figure S2 for more details) and (10). Finally, we used Eq. (10) to determine the salt polarizability.

In Section IV, we also list the values of salt polarizabilities calculated using the DE method as commonly used in earlier literature. For this method, we first calculated the polarizability values through Eqs. (1) and (2) at different concentrations for dilute solutions of a certain kind of salt. We then plot graphs of polarizability $\alpha_{\text {salt }} v s . c_{\text {salt }}(\mathrm{mol} / \mathrm{L})$ and extrapolate to the infinite dilution limit (see Figure S1 for more details).

\section{RESULTS AND DISCUSSIONS}

Salt polarizability $\alpha_{\text {salt }}$. The polarizabilities $\left(\alpha_{\text {salt }}\right)$ of 32 common salts in aqueous solution were determined from Eq. (10) using the experimentally obtained values of $K$ and $\gamma^{\prime}$. In Table 1 , we list the salt polarizabilities at $25^{\circ} \mathrm{C}$ and $37^{\circ} \mathrm{C}$ (denoted as $\alpha_{25}$ and $\alpha_{37}$, respectively) as determined using our method, together with the measured values of $K$ and $\gamma^{\prime}$ at $25^{\circ} \mathrm{C}$. The $\alpha_{37}$ data are included for potential use in life science research. The percent errors range from $0.499 \%-3.75 \%$ for $\alpha_{25}$ and $0.433 \%-4.10 \%$ for $\alpha_{37}$, with average percent errors of $1.62 \%$ and $1.58 \%$ for $\alpha_{25}$ and $\alpha_{37}$, respectively.

Furthermore, we compare our results for $\alpha_{25}$ with values obtained from earlier literature. Extensive data sets on salt polarizabilities are available as determined by Pyper et al. $\left(\alpha^{\mathrm{P}}\right)^{19}$ and Heydweiller $\left(\alpha^{\mathrm{H}}\right) \cdot{ }^{25}$ We note that there exist large discrepancies between these two previous sets of data, with polarizabilities for the same salt differing by as much as $40 \%$ (for example, see entries for $\mathrm{LiCl}, \mathrm{NaBr}, \mathrm{KI}$ ), which highlights the importance of more accurate determination of salt polarizabilities. (Note that the data in Ref. ${ }^{25}$ were taken at $18^{\circ} \mathrm{C}$ instead of $25^{\circ} \mathrm{C}$. However, from the temperature dependence of salt polarizabilities to be discussed later, a $7^{\circ} \mathrm{C}$ difference in temperature will increase the polarizabilities by no more than $1 \%$ for most salts.) Comparing our $\alpha_{25}$ with $\alpha^{\mathrm{P}}$ and $\alpha^{\mathrm{H}}$, we find that our data generally lie between these two previous data sets, closer to the data from Heydweiller. ${ }^{25}$ Moreover, the 
Table 1: $K$ and $\gamma^{\prime}$ in Eq. (10) as well as the Salt Polarizability in Aqueous Solutions

\begin{tabular}{|c|c|c|c|c|c|c|c|}
\hline salt & $K_{25}$ & $\gamma_{25}^{\prime}$ & $\alpha_{25}{ }^{a}$ & $\alpha_{25}^{\mathrm{P} b}$ & $\alpha_{18}^{\mathrm{H} c}$ & $\alpha_{25}^{\mathrm{DE} d}$ & $\alpha_{37^{a}}$ \\
\hline $\mathrm{NaF}$ & 0.286 & -0.149 & $0.932 \pm 0.030$ & & & 0.718 & $0.927 \pm 0.038$ \\
\hline $\mathrm{KF}$ & 0.261 & 0.658 & $2.017 \pm 0.071$ & 1.280 & 1.930 & 1.936 & $1.999 \pm 0.042$ \\
\hline $\mathrm{CsF}$ & 0.431 & 1.600 & $4.086 \pm 0.088$ & & & 3.908 & $3.935 \pm 0.057$ \\
\hline $\mathrm{LiCl}$ & 0.464 & 0.954 & $3.271 \pm 0.033$ & 2.262 & 3.462 & 3.296 & $3.224 \pm 0.057$ \\
\hline $\mathrm{NaCl}$ & 0.511 & 1.004 & $3.532 \pm 0.034$ & 2.391 & 3.65 & 3.266 & $3.359 \pm 0.030$ \\
\hline $\mathrm{KCl}$ & 0.489 & 1.467 & $4.126 \pm 0.024$ & 2.931 & 4.465 & 4.304 & $4.080 \pm 0.043$ \\
\hline $\mathrm{RbCl}$ & 0.546 & 1.785 & $4.824 \pm 0.084$ & 3.336 & 5.044 & 4.742 & $4.822 \pm 0.081$ \\
\hline $\mathrm{CsCl}$ & 0.689 & 2.113 & $5.881 \pm 0.030$ & 4.063 & 6.056 & 5.977 & $5.834 \pm 0.069$ \\
\hline $\mathrm{MgCl}_{2}$ & 1.059 & 1.030 & $5.775 \pm 0.091$ & & 6.551 & 5.812 & $5.690 \pm 0.079$ \\
\hline $\mathrm{CaCl}_{2}$ & 1.283 & 1.315 & $7.094 \pm 0.122$ & 4.868 & & 7.179 & $6.722 \pm 0.095$ \\
\hline $\mathrm{SrCl}_{2}$ & 1.446 & 1.124 & $7.466 \pm 0.076$ & 5.178 & 7.812 & 7.330 & $7.385 \pm 0.076$ \\
\hline $\mathrm{BaCl}_{2}$ & 1.575 & 1.525 & $8.576 \pm 0.093$ & 5.830 & 8.724 & 8.454 & $8.498 \pm 0.156$ \\
\hline $\mathrm{LiBr}$ & 0.637 & 1.531 & $4.817 \pm 0.154$ & & 4.862 & 4.753 & $4.747 \pm 0.141$ \\
\hline $\mathrm{NaBr}$ & 0.744 & 1.320 & $4.935 \pm 0.048$ & 3.276 & 5.104 & 4.852 & $4.873 \pm 0.046$ \\
\hline $\mathrm{KBr}$ & 0.710 & 1.880 & $5.621 \pm 0.065$ & 3.820 & 5.929 & 5.627 & $5.525 \pm 0.064$ \\
\hline $\mathrm{CsBr}$ & 0.845 & 2.673 & $7.333 \pm 0.086$ & & 7.511 & 7.402 & $7.296 \pm 0.074$ \\
\hline $\mathrm{MgBr}_{2}$ & 1.585 & 1.924 & $9.203 \pm 0.124$ & & 9.498 & 8.760 & $9.119 \pm 0.082$ \\
\hline $\mathrm{NaI}$ & 1.104 & 2.044 & $7.447 \pm 0.065$ & 4.842 & 7.666 & 7.231 & $7.443 \pm 0.064$ \\
\hline KI & 1.094 & 2.494 & $8.070 \pm 0.048$ & 5.391 & 8.546 & 8.121 & $8.057 \pm 0.030$ \\
\hline CsI & 1.248 & 3.253 & $9.809 \pm 0.080$ & & & 9.682 & $9.753 \pm 0.100$ \\
\hline $\mathrm{LiNO}_{3}$ & 0.426 & 1.590 & $4.054 \pm 0.080$ & & 4.231 & 4.076 & $4.000 \pm 0.100$ \\
\hline $\mathrm{NaNO}_{3}$ & 0.457 & 1.681 & $4.313 \pm 0.089$ & 2.870 & 4.457 & 4.180 & $4.195 \pm 0.048$ \\
\hline $\mathrm{KNO}_{3}$ & 0.450 & 2.139 & $4.960 \pm 0.039$ & 3.407 & 5.258 & 4.688 & $4.862 \pm 0.034$ \\
\hline $\mathrm{Mg}\left(\mathrm{NO}_{3}\right)_{2}$ & 1.114 & 2.080 & $7.542 \pm 0.056$ & & 8.082 & 7.575 & $7.475 \pm 0.105$ \\
\hline $\mathrm{LiClO}_{4}$ & 0.330 & 2.433 & $4.909 \pm 0.071$ & 3.402 & & 4.867 & $4.891 \pm 0.076$ \\
\hline $\mathrm{NaClO}_{4}$ & 0.383 & 2.407 & $5.049 \pm 0.050$ & 3.541 & 5.370 & 5.021 & $4.956 \pm 0.070$ \\
\hline $\mathrm{Ba}\left(\mathrm{ClO}_{4}\right)_{2}$ & 1.237 & 4.548 & $11.67 \pm 0.12$ & 8.138 & & 12.241 & $11.65 \pm 0.132$ \\
\hline $\mathrm{Li}_{2} \mathrm{SO}_{4}$ & 0.892 & 0.639 & $4.528 \pm 0.052$ & 3.734 & 5.544 & 5.292 & $4.456 \pm 0.104$ \\
\hline $\mathrm{Na}_{2} \mathrm{SO}_{4}$ & 1.015 & 0.659 & $4.907 \pm 0.083$ & 3.978 & 5.921 & 4.932 & $4.804 \pm 0.103$ \\
\hline $\mathrm{K}_{2} \mathrm{SO}_{4}$ & 1.008 & 1.474 & $6.224 \pm 0.128$ & & & 6.806 & $6.170 \pm 0.151$ \\
\hline $\mathrm{Cs}_{2} \mathrm{SO}_{4}$ & 1.345 & 2.775 & $9.462 \pm 0.231$ & & & 10.16 & $9.458 \pm 0.108$ \\
\hline $\mathrm{MgSO}_{4}$ & 1.197 & -0.097 & $4.670 \pm 0.075$ & & & 4.404 & $4.484 \pm 0.119$ \\
\hline
\end{tabular}

${ }^{a}$ The salt polarizability obtained by our method (with the standard error after \pm ). ${ }^{b}$ The salt polarizability obtained by Pyper. ${ }^{19 c}$ The salt polarizability obtained by Heydweiller. ${ }^{25}$ ${ }^{d}$ The salt polarizability obtained by DE method using our experimental data. The units of the polarizabilities are $\AA^{3}$ and the subscripts refer to the temperature in ${ }^{\circ} \mathrm{C}$. The conversion $\alpha(\mathrm{SI}) / \mathrm{C} \mathrm{m}^{2} \mathrm{~V}^{-1}=4 \pi \varepsilon_{0} \times 10^{-6} \alpha(\mathrm{cgs}) / \mathrm{cm}^{3}$ is to be used if SI unit is desired. 
error bars in our data are much smaller compared to the differences between these two data sets. Therefore, we believe our method is more accurate for determining salt polarizabilities and therefore the values we report for $\alpha_{\text {salt }}$ should be more reliable than previous literature values.

In earlier studies, ${ }^{25}$ the apparent salt polarizability (or molar refractivity) was first measured as a function of molar salt concentration using Eqs. (1) and (2), and then extrapolated to zero concentration to obtain its value at infinite dilution. Pyper et al. obtained what they termed the "total in-solution molar polarizability" by the DE method. ${ }^{19}$ However, the apparent salt polarizability generally varies nonlinearly with concentration, with large error bars in the low concentration range. Therefore the DE method introduces significant uncertainties in the value of salt polarizability at infinite dilution (see Figure S1). In contrast, our method depends on the determination of refractive indices and volumes, both of which can be easily and accurately measured experimentally. The linear variation of the refractive index in the low concentration, with a slope derived in our earlier work, ${ }^{28}$ allows straightforward and accurate determination of the salt polarizability; Figure 1 shows the quality of the linear fitting for the refractive index in the low concentration range. In order to demonstrate the superiority of our new method, we list the salt polarizability values obtained by direct extrapolation $\left(\alpha_{25}^{\mathrm{DE}}\right)$ from our experimental data. While values of $\alpha_{25}^{\mathrm{DE}}$ are generally more accurate than previous literature values, there are a few cases (such as for $\mathrm{KF}$ and $\mathrm{MgSO}_{4}$ ), where the values obtained from direct extrapolation contain significant errors in reference to the data obtained using the slope of the refractive index.

We used six different salts ( $\mathrm{LiCl}, \mathrm{NaCl}, \mathrm{LiBr}, \mathrm{NaBr}, \mathrm{MgSO}_{4}$ and $\mathrm{MgBr}_{2}$ ), each at six different temperatures $\left(25^{\circ} \mathrm{C}, 35^{\circ} \mathrm{C}, 37^{\circ} \mathrm{C}, 45^{\circ} \mathrm{C}, 55^{\circ} \mathrm{C}\right.$ and $\left.65^{\circ} \mathrm{C}\right)$ to examine the effect of temperature on the salt polarizability. Figure 2(a) shows that the temperature dependence of salt polarizability is generally quite weak. The trend is more clear when we plot the ratio $\alpha_{\text {salt }} / \alpha_{25}$ in the range between 0.94 and 1 in Figure 2(b). $\alpha_{\text {salt }}$ for all six salts decreases with increasing temperature. The physical origin of this decrease is not clear. Also, while at a 


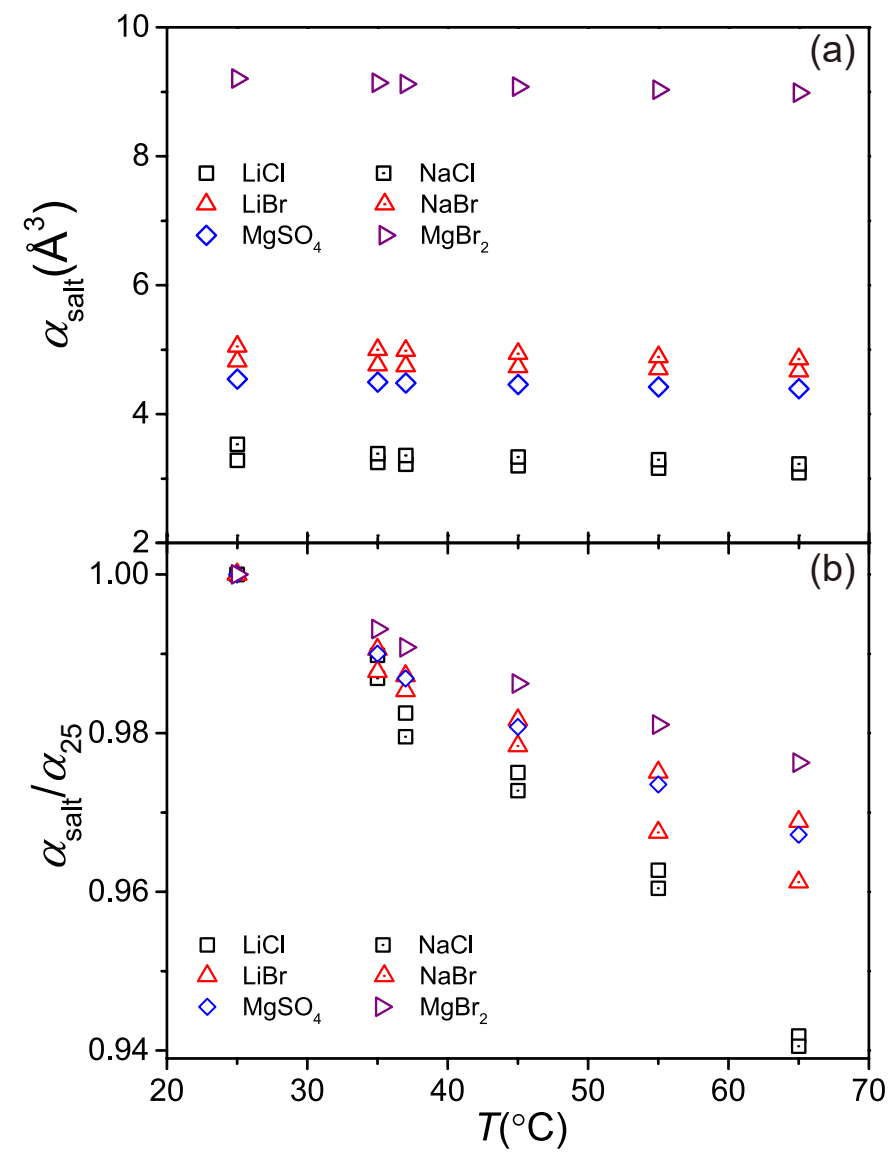

Figure 2: The temperature dependence of (a) the salt polarizability $\alpha_{\text {salt }}$ and (b) the relative polarizability $\alpha_{\text {salt }} / \alpha_{25}$.

given temperature, the relative magnitude of the salts polarizabilities reflects the dominance of the anions (due to its larger volume and more extensive electron cloud), no simple trend can be discerned in the rate of the temperature change of the salt polarizabilities - the differences in the slope in Figure 2(b) are primarily due to the differences in the magnitude of the salt polarizabilities.

Ion polarizability $\alpha_{\text {ion }}$. To date, there is no established method for obtaining single-ion polarizabilities in aqueous solutions. In our previous work, ${ }^{28}$ we found that the refractive index of an aqueous mixture is largely determined by the polarizability of the ions and the rate of change of the solution volume with addition of salt. At salt concentrations approaching infinite dilution, all ions are completely separated from each other. ${ }^{23}$ Therefore, the salt polarizability $\alpha_{\text {salt }}$ measured at the infinite dilution limit is the sum of the polarizabilities of 
the constituting cations and anions.

Table 2: The Bare Ionic Radius, as well as Ion Polarizabilities in Different Phases

\begin{tabular}{cccccccc}
\hline Ion & $r_{\text {bare }}{ }^{a}$ & $\alpha_{\text {ion(gas })^{b}}$ & $\alpha_{\text {ion(crys })^{c}}$ & $\alpha_{\text {ion(aq) }}^{\mathrm{P}}$ & $\alpha_{\text {ion(aq) }}^{\mathrm{H}}$ & $\alpha_{\text {ion(aq) }}^{\mathrm{M}}$ & $\alpha_{\text {ion(aq) }}{ }^{g}$ \\
\hline $\mathrm{Li}^{+}$ & 0.94 & 0.032 & 0.03 & 0.019 & 0.029 & 0.029 & 0.029 \\
$\mathrm{Na}^{+}$ & 1.17 & 0.157 & 0.41 & 0.100 & 0.217 & 0.18 & $0.279 \pm 0.001$ \\
$\mathrm{~K}^{+}$ & 1.49 & 0.830 & 1.33 & 0.534 & 1.032 & 0.81 & $0.873 \pm 0.009$ \\
$\mathrm{Rb}^{+}$ & 1.63 & 1.370 & 1.98 & 0.905 & 1.611 & 1.32 & $1.571 \pm 0.051$ \\
$\mathrm{Cs}^{+}$ & 1.86 & 2.360 & 3.34 & 1.528 & 2.623 & 2.02 & $2.628 \pm 0.014$ \\
$\mathrm{Ca}^{2+}$ & 1.00 & 0.490 & 1.10 & 0.319 & & 0.44 & $0.588 \pm 0.056$ \\
$\mathrm{Sr}^{2+}$ & 1.13 & 0.870 & 1.60 & 0.520 & 0.946 & 0.81 & $0.961 \pm 0.010$ \\
$\mathrm{Ba}^{2+}$ & 1.98 & 1.560 & 2.50 & 1.010 & 1.858 & & $2.070 \pm 0.027$ \\
$\mathrm{~F}^{-}$ & 1.16 & 2.467 & 0.64 & 0.88 & 0.898 & 1.3 & $1.144 \pm 0.064$ \\
$\mathrm{Cl}^{-}$ & 1.64 & 5.482 & 2.96 & 2.54 & 3.433 & 3.5 & $3.253 \pm 0.033$ \\
$\mathrm{Br}^{-}$ & 1.80 & 7.268 & 4.16 & 3.42 & 4.897 & 4.6 & $4.748 \pm 0.056$ \\
$\mathrm{I}^{-}$ & 2.05 & 10.275 & 6.43 & 5.00 & 7.514 & 7.5 & $7.197 \pm 0.039$ \\
$\mathrm{ClO}_{4}^{-}$ & & & & 3.675 & 5.153 & & $4.825 \pm 0.071^{h}$ \\
$\mathrm{NO}_{3}^{-}$ & 1.79 & & 3.81 & 3.011 & 4.221 & & $4.049 \pm 0.088^{i}$ \\
$\mathrm{SO}_{4}^{2-}$ & & & 4.280 & 5.486 & & $4.432 \pm 0.110^{j}$ \\
\hline
\end{tabular}

${ }^{a}$ The bare ionic radius with units of $\AA .{ }^{b}$ The ion polarizability in gas-phase. ${ }^{c}$ The ion polarizability in crystal-phase. ${ }^{d}$ The ion polarizability in aqueous solution obtained by the direct extrapolation method at $25^{\circ} \mathrm{C} ;{ }^{19}$ e The ion polarizability in aqueous solution obtained by the direct extrapolation method at $18^{\circ} \mathrm{C} .{ }^{f}$ The ion polarizability in aqueous solution obtained by DFT method. ${ }^{18} \mathrm{~g}$ The ion polarizability in aqueous solution obtained from our method (with the standard error after \pm ) at $25^{\circ} \mathrm{C}$. ${ }^{h}$ The polarizabilities of $\mathrm{ClO}_{4}^{-}$ in $\mathrm{LiClO}_{4}$ and $\mathrm{NaClO}_{4}$ are $4.880 \pm 0.071$ and $4.770 \pm 0.049$, respectively. ${ }^{i}$ The polarizabilities of $\mathrm{NO}_{3}^{-}$in $\mathrm{LiNO}_{3}, \mathrm{NaNO}_{3}$ and $\mathrm{KNO}_{3}$ are $4.025 \pm 0.080,4.034 \pm 0.088$ and $4.087 \pm 0.030$, respectively. ${ }^{j}$ The polarizabilities of $\mathrm{SO}_{4}^{2-}$ in $\mathrm{Li}_{2} \mathrm{SO}_{4}, \mathrm{Na}_{2} \mathrm{SO}_{4}$, and $\mathrm{K}_{2} \mathrm{SO}_{4}$ are $4.470 \pm 0.052,4.349 \pm 0.081$, and $4.478 \pm 0.110$, respectively. The units of the polarizabilities are $\AA^{3}$.

In order to obtain the polarizability of a single ion in water, a reference polarizability value needs to be defined. Earlier work ${ }^{15,18,19,33}$ suggested that the polarizabilities of small cations are largely unaffected by the aqueous environment. In addition, the smaller value of the polarizability of smaller cations makes them appropriate choices as references since this will minimize the errors - the errors will be bounded by the value of the polarizability of the reference ion. In view of these considerations, the $\mathrm{Li}^{+}$ion constitutes the best choice as the reference, being the smallest ion involved in our measurements. According to density 
functional theory (DFT), the polarizability of $\mathrm{Li}^{+}$is $0.029 \AA^{3},{ }^{18}$ and we set this value as our reference polarizability.

Once the reference polarizability is selected, all $\alpha_{\text {ion }}$ values can be deduced. The process for calculating the polarizabilities of ions is as follows: First, $\alpha_{\mathrm{Cl}^{-}}$is determined by subtracting $\alpha_{\mathrm{Li}^{+}}$from $\alpha_{\mathrm{LiCl}}$. Next, the polarizabilities of other metal ions $\left(\alpha_{\mathrm{Na}^{+}}, \alpha_{\mathrm{K}^{+}}, \alpha_{\mathrm{Rb}^{+}}, \alpha_{\mathrm{Cs}^{+}}\right.$, $\alpha_{\mathrm{Ca}^{2+}}, \alpha_{\mathrm{Sr}^{2+}}$ and $\left.\alpha_{\mathrm{Ba}^{2+}}\right)$ are determined by subtracting $\alpha_{\mathrm{Cl}^{-}}$from the polarizabilities of the corresponding chloride salts. In an analogous manner, the polarizabilities of other anions $\left(\alpha_{\mathrm{F}^{-}}, \alpha_{\mathrm{Br}^{-}}\right.$and $\left.\alpha_{\mathrm{I}^{-}}\right)$are obtained by subtracting $\alpha_{\mathrm{K}^{+}}$polarizabilities of the corresponding potassium chlorides. Finally, the polarizabilities of polyatomic anions are determined from their salts by subtracting the cation polarizabilities. While theoretically the polarizability of the polyanions should not depend on the identity of the metal counterions, experimental uncertainties and finite concentration effects can result in appreciably different values for the anion depending on the cation identity. We report the polyatomic anion polarizabilities based on the average from several salts, with the actual values for the different salts indicated in the caption of Table 2. The polarizability of $\mathrm{Mg}^{2+}$ is not listed in Table 2 since the method outlined above yields a negative $\alpha_{\mathrm{Mg}^{2+}}\left(-0.730 \AA^{3}\right.$ from using $\mathrm{MgCl}_{2}$ and $-0.299 \AA^{3}$ from using $\left.\mathrm{MgBr}_{2}\right)$. This pathological behavior may originate from the following two sources: First, complete removal of water from these highly hygroscopic magnesium salts is very difficult; moreover these two salts easily decompose at high temperatures (as a way to remove water), making an accurate determination of the mass difficult. Second, $\alpha_{\mathrm{Mg}^{2+}}=0.08 \AA^{3}$ is quite small according to the DFT estimate. ${ }^{18}$ However, the standard errors of anions such as $\alpha_{\mathrm{Cl}^{-}}$and $\alpha_{\mathrm{Br}^{-}}$are quite large, and one $\mathrm{Mg}^{2+}$ corresponds to two $\mathrm{Cl}^{-} / \mathrm{Br}^{-}$counterions. Therefore, the errors of $\alpha_{\mathrm{Mg}^{2+}}$ from $\alpha_{\mathrm{MgCl}_{2}}-2 \alpha_{\mathrm{Cl}^{-}}$and $\alpha_{\mathrm{MgBr}_{2}}-2 \alpha_{\mathrm{Br}^{-}}$can exceed $\alpha_{\mathrm{Mg}^{2+}}$ itself.

The ion polarizabilities in the aqueous state $\alpha_{\text {ion(aq) }}$ at $25^{\circ} \mathrm{C}$ obtained from our measurements are listed in Table 2. The average standard error in $\alpha_{\text {ion(aq) }}$ is 0.041 , ranging from 0.007 to 0.079 . For comparison, we provide ion polarizability values from previous studies: 
those obtained from DE experimental measurements by Pyper et al. ${ }^{19}$ (denoted as $\alpha_{\mathrm{ion}(\mathrm{aq})}^{\mathrm{P}}$ ) and those in the aqueous state computed from first-principles by Molina et al. ${ }^{18}$ (denoted as $\left.\alpha_{\mathrm{ion}(\mathrm{aq})}^{\mathrm{M}}\right)$. In addition, using $\alpha_{\mathrm{Li}^{+}}=0.029 \AA^{3}$ as the reference polarizability, we have calculated the ion polarizability values (denoted as $\left.\alpha_{\text {ion(aq) }}^{\mathrm{H}}\right)$ using Heydweiler's molar refractivity data. ${ }^{25}$ Our results are in closer agreement with the theoretical values by Molina et al. than previous results from Heydweiler ${ }^{25}$ and Pyper et al. ${ }^{19}$ For most ions, our results and those reported by Molina et al. agree to within a $10 \%$ difference. We believe this closer agreement between our results and those of Molina et al. ${ }^{18}$ most likely reflect the improved accuracy and reliability of our results relative to previous experimental data.

Factors affecting the ion polarizability $\alpha_{\text {ion }}$. Since polarizability has unit of volume, it is natural to expect that it will scale with the volume of the ion. In Figure 3, we plot the ion polarizability vs. the cube of the bare ionic radius $r_{\text {bare }}^{3}$ in the aqueous, gaseous, and crystalline phases, where $r_{\text {bare }}$ is the radius of an individual (non-hydrated) ion. For monovalent ions of the alkali metal series and the halogen series, we indeed find that the polarizability in the aqueous, crystalline and gaseous states is linearly proportional to $r_{\text {bare }}^{3}$. This result is in agreement with the findings by Iwadate et al., who reported a linear relationship between $\alpha_{\text {ion }}$ and $r_{\text {bare }}^{3}$ in ionic melts, ${ }^{33}$ and also corroborates the theories by Machmer ${ }^{35}$ who proposed a linear relationship between $\alpha_{\text {ion }}$ and $r_{\text {bare }}^{3}$ for spherical particles. However, the linear relationship between ion polarizability and $r_{\text {bare }}^{3}$ does not hold for monatomic divalent and multivalent ions. The reason for the deviation from linearity requires further study, but we suspect that the stronger interactions between the ions and the surrounding water molecules for ions with high charges may possibly distort the spherical shape in the charge density distribution on and around the ions.

From Figure 3 we can also compare the ion polarizabilities in the different states of matter. We find that for most ions the aqueous phase polarizability generally lies between their values in the crystalline and gaseous phases. The polarizability of cations generally increases going from the gaseous phase to the crystalline phase, probably due to the stronger shielding of the 


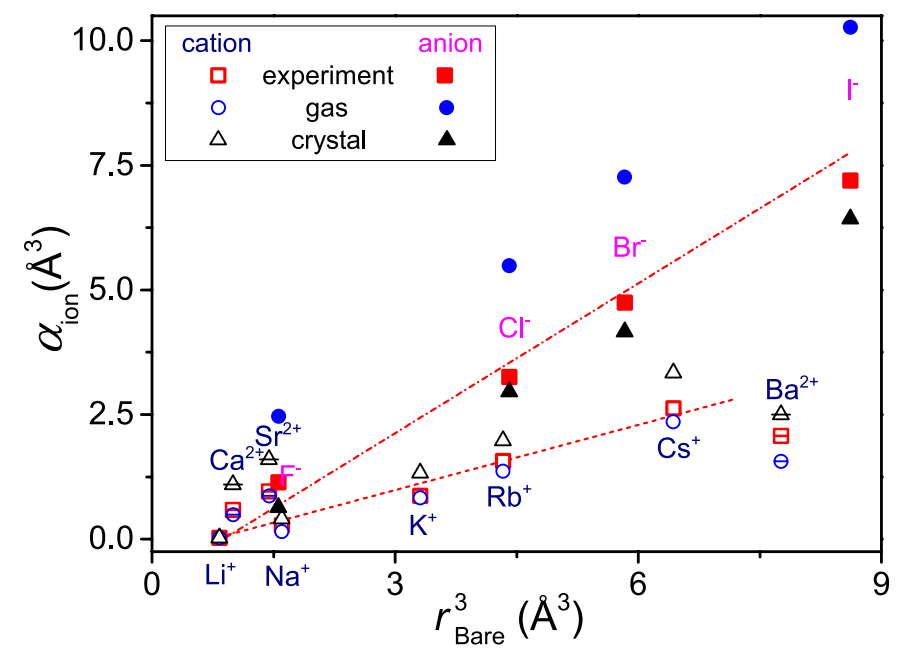

Figure 3: The ion polarizability $\alpha_{\text {ion }}$ as a relationship of the cubic bare ionic radius $r_{\text {bare }}^{3}$ in three different states (solution, gas and crystal). The horizontal segment inside the symbol stands for the divalent cations.

electrostatic field by the surrounding species in the more condensed phases. ${ }^{19}$ However, we note that the cation polarizabilities in the aqueous phase are closer to their gaseous-phase values than to their crystalline-phase values, suggesting that the water molecules surrounding cations only exert a minimal shielding effect on the electric field. The opposite trend is observed for anions in the halogen series, with the ion polarizability decreasing from the gaseous phase to the crystalline phase. Similar trends were seen in other studies. ${ }^{15,18,19,22}$ However, there is no clear theoretical argument from past literature to explain how the aqueous environment reduces the anion polarizability from its gas-phase value. Qualitatively, the reduction in polarizability in the aqueous phase relative to the gaseous phase could be due to the overlap between the electron cloud of the anions and those of the solvent molecules, which might reduce the ability of the anions to develop induced dipoles. ${ }^{40}$

Next, we investigate the relationship between the atomic number (number of protons) on the ion polarizability. Based on data for halogen salts with heavy metal ions, Krishnamur$\mathrm{ti}^{36,37}$ suggested that the polarizabilities of the halogen ions are quadratic in their atomic number $N$. To test this relationship, in Figure 4, we plot the ion polarizability as a function of the square of the atomic number $N^{2}$. For the larger ions in the same family, there indeed 


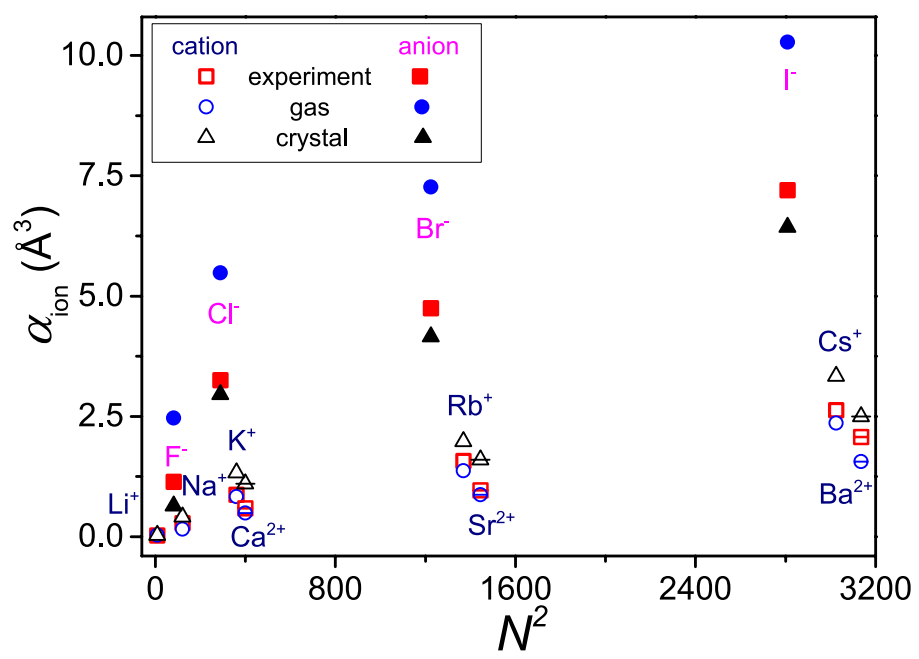

Figure 4: The ion polarizability $\alpha_{\text {ion }}$ as a relationship of the square atomic number $N^{2}$ in three different states (solution, gas and crystal). The horizontal segment inside the symbol stands for the divalent cations.

seem to be a reasonable linear relation between $\alpha_{\text {ion }}$ and $N^{2}$, but with different offsets for the different ion families. However, for small ions such as $\mathrm{Li}^{+}, \mathrm{Na}^{+}$and $\mathrm{F}^{-}$, the relationship clearly does not hold.

Summarizing the results presented in Figures. 3 and 4, it is clear that both the volume and the atomic number of the ions play a role in determining their polarizabilities However, except for the simple alkali metal and halogen ions, the relationship between these attributes and the polarizability is not a simple one. Further theoretical studies are clearly needed.

\section{CONCLUSIONS}

This work presents data on salt and ion polarizabilities in aqueous solutions based on a new extrapolation method using the refractive index and volume measurements. Previously, DE method was used to determine salt polarizabilities via refractive indices and mass density. However, in the experimentally accessible range of concentration, direct extrapolation can result in significant inaccuracies due to the non-linear relationship between the apparent salt polarizability and salt concentration (see Figure S1). In this work, we calculated the salt 
polarizabilities of 32 strong electrolytes at the infinite dilution limit by using the theoretical limiting slope in the relationship between the refractive index and salt concentration derived in our earlier work. ${ }^{28}$ This theoretical slope is linearly dependent on the salt polarizability and involves the density and refractive index of pure water, as well as the limiting rate of change of the volume of an aqueous solution with salt concentration which is obtained by taking into account the nonlinear corrections due to the Debye-Hückel limiting law in the volume data. By setting $\mathrm{Li}^{+}$as the reference, we were able to obtain the polarizabilities of the constituting ions. Compared to previous experimental data, our results are in better agreement with the theoretical results obtained from density functional theory of Molina et $a l .{ }^{18}$ We believe this closer agreement between our results and those of Molina et al. ${ }^{18}$ is validation of the theoretical results and also reflects the improved accuracy and reliability of our results.

We have also studied several key factors influencing salt and ion polarizability - the temperature, the bare ionic radius, and the atomic number. We found that there is a slight decrease in the salt polarizability with increasing temperature. We found that the the linear relationship between ion polarizability and the cube of the bare ionic radius applies to univalent ions but not to divalent ions. For the dependence on the atomic number, our results showed that for divalent ions and large monovalent ions, there is an approximate linear relationship between the polarizability and the square of the atomic number, as suggested by Krishnamurti. ${ }^{36,37}$ But this relationship does not hold for small monovalent ions. The physical origins of these dependences require further study.

\section{Acknowledgement}

This work was supported by the National Natural Science Foundation of China (Grant Nos. 21674113 and 21334007) and the International Partnership Program of CAS (Grant No. 121522KYSB20160015). Y.L. acknowledges the Youth Innovation Promotion Association of 
CAS (Grant No. 2016204) for financial support. B.Z. acknowledges the A-STAR fellowship for financial support.

\section{Supporting Information Available}

The Supporting Information provides the relationship between polarizability $\alpha_{25}^{\mathrm{DE}}$ and concentration $c_{\text {salt }}(\mathrm{mol} / \mathrm{L})$ for $\mathrm{KF}, \mathrm{NaCl}, \mathrm{NaNO}_{3}, \mathrm{CaCl}_{2}, \mathrm{Li}_{2} \mathrm{SO}_{4}$, and $\mathrm{MgSO}_{4}$; the relationship between volume ratio $V_{s s} / V_{s}$ and salt mole fraction $x_{\text {salt }}$ for $\mathrm{KF}, \mathrm{NaCl}, \mathrm{NaNO}_{3}, \mathrm{CaCl}_{2}$, $\mathrm{Li}_{2} \mathrm{SO}_{4}$, and $\mathrm{MgSO}_{4}$ at $25^{\circ} \mathrm{C}$.

\section{References}

(1) Griffiths, D. J. Introduction to Electrodynamics, 3rd ed.; Pearson Education: Dorling Kindersley, 2007.

(2) Lagi, M.; Lo Nostro, P.; Fratini, E.; Ninham, B. W.; Baglioni, P. Insights into Hofmeister Mechanisms: Anion and Degassing Effects on the Cloud Point of Dioctanoylphosphatidylcholine/Water Systems. J. Phys. Chem. B 2007, 111, 589-597.

(3) Cheng, J.; Vecitis, C. D.; Hoffmann, M. R.; Colussi, A. J. Experimental Anion Affinities for the Air/Water Interface. J. Phys. Chem. B 2006, 110, 25598-25602.

(4) Jungwirth, P.; Tobias, D. J. Specific Ion Effects at the Air/Water Interface. Chem. Rev. 2006, 106, 1259-1281.

(5) Lo Nostro, P.; Ninham, B. W. Hofmeister Phenomena: An Update on Ion Specificity in Biology. Chem. Rev. 2012, 112, 2286-2322.

(6) Kunz, W.; Henle, J.; Ninham, B. W. 'Zur Lehre von der Wirkung der Salze' (about the Science of the Effect of Salts): Franz Hofmeister's Historical Papers. Curr. Opin. Colloid Interface Sci. 2004, 9, 19-37. 
(7) Zhang, Y.; Furyk, S.; Bergbreiter, D. E.; Cremer, P. S. Specific Ion Effects on the Water Solubility of Macromolecules: PNIPAM and the Hofmeister Series. J. Amer. Chem. Soc. 2005, 127, 14505-14510.

(8) Ninham, B. W.; Yaminsky, V. Ion Binding and Ion Specificity: the Hofmeister Effect and Onsager and Lifshitz Theories. Langmuir 1997, 13, 2097-2108.

(9) Ninham, B. W.; Lo Nostro, P. Molecular Forces and Self Assembly; Cambridge University Press: Cambridge, 2010.

(10) Jungwirth, P.; Tobias, D. J. Molecular Structure of Salt Solutions: A New View of the Interface with Implications for Heterogeneous Atmospheric Chemistry. J. Phys. Chem. B 2001, 105, 10468-10472.

(11) dos Santos, A. P.; Levin, Y. Ions at the Water-Oil Interface: Interfacial Tension of Electrolyte Solutions. Langmuir 2011, 28, 1304-1308.

(12) Levin, Y. Polarizable Ions at Interfaces. Phys. Rev. Lett. 2009, 102, 147803.

(13) Levin, Y.; Dos Santos, A. P. Ions at the Air-Water Interface: An End to a HundredYear-Old Mystery? Phys. Rev. Lett. 2009, 103, 257802.

(14) Soniat, M.; Rick, S. W. Charge Transfer Effects of Ions at the Liquid Water/Vapor Interface. J. Chem. Phys. 2014, 140, 184703.

(15) Pauling, L. The Theoretical Prediction of the Physical Properties of Many-Electron Atoms and Ions. Mole Refraction, Diamagnetic Susceptibility, and Extension in Space. Proc. R. Soc. London 1927, 114, 181-211.

(16) Lide, D. R.; Mickey, W. CRC Handbook of Chemistry and Physics, 90th ed.; CRC Press: London, 2010. 
(17) Bauer, B. A.; Lucas, T. R.; Krishtal, A.; Alsenoy, C. V.; Patel, S. Variation of Ion Polarizability from Vacuum to Hydration: Insights from Hirshfeld Partitioning. J. Phys. Chem. A 2010, 114, 8984-8992.

(18) Molina, J. J.; Lectez, S.; Tazi, S.; Salanne, M.; Dufreche, J. -F.; Roques, J.; Simoni, E.; Madden, P. A.; Turq, P. Ions in Solutions: Determining Their Polarizabilities from First-Principles. J. Chem. Phys. 2011, 134, 014511.

(19) Pyper, N. C.; Pike, C. G.; Edwards, P. P. The Polarizabilities of Species Present in Ionic Solutions. Mol. Phys. 1992, 76, 353-372.

(20) Masia, M. Estimating Chloride Polarizability in a Water Solution. J. Phys. Chem. A 2013, 117, 3221-3226.

(21) Jungwirth, P.; Curtis, J. E.; Tobias, D. J. Polarizability and Aqueous Solvation of the Sulfate Dianion. Chem. Phys. Lett. 2003, 367, 704-710.

(22) Jungwirth, P.; Tobias, D. J. Chloride Anion on Aqueous Clusters, at the Air-Water Interface, and in Liquid Water: Solvent Effects on $\mathrm{Cl}^{-}$Polarizability. J. Phys. Chem. A 2002, 106, 379-383.

(23) Feynman, R. P.; Leighton, R. B.; Sands, M. The Feynman Lectures on Physics, Vol. II: New Millennium Edition: Mainly Electromagnetism and Matter; Basic Books: New York, 2010.

(24) Fajans, K. The Refraction and Dispersion of Gases and Vapours. Z. Phys. Chem. B 1934, 24, 103-154.

(25) Heydweiller, A. Optische Untersuchungen an Wasserigen Elektrolytlosungen. Phys. Z. 1925, 26, 526-556.

(26) Bauer, N.; Fajans, K. The Molar Dispersion and Refraction of Free and Bonded Ions. J. Amer. Chem. Soc. 1942, 64, 3023-3034. 
(27) Le Fevre, R. J. W. Molecular Refractivity and Polarizability. Adv. Phys. Org. Chem. 1965, 3, 1-90.

(28) An, N.; Zhuang, B.; Li, M.; Lu, Y.; Wang, Z. -G. Combined Theoretical and Experimental Study of Refractive Indices of Water-Acetonitrile-Salt System. J. Phys. Chem. B 2015, 119, 10701-10709.

(29) Millero, F. J. Partial Molal Volume of Ions in Various Solvents. J. Phys. Chem. 1969, 73, 2417-2420.

(30) Millero, F. J.; Laferriere, A. L.; Chetirkin, P. V. The Partial Molal Volumes of Electrolytes in $0.725 \mathrm{~m}$ Sodium Chloride Solutions at 25ㄷ. J. Phys. Chem. 1977, 81, 17371745.

(31) Iwadate, Y.; Kawamura, K.; Murakami, K.; Igarashi, K.; Mochinaga, J. Electronic Polarizabilities of $\mathrm{Tl}^{+}, \mathrm{Ag}^{+}$, and $\mathrm{Zn}^{2+}$ Ions Estimated from Refractive Index Measurements of $\mathrm{TlNO}_{3}, \mathrm{AgNO}_{3}$, and $\mathrm{ZnCl}_{2}$ melts. J. Chem. Phys. 1982, 77, 6177-6183.

(32) Shanker, J.; Agrawal, H. B. Electronic Polarizabilities, Ionic Radii, and Repulsive Potential Parameters for Diatomic Alkali Halide Molecules. Can. J. Phys. 1980, 58, 950-956.

(33) Iwadate, Y.; Mochinaga, J.; Kawamura, K. Refractive Indexes of Ionic Melts. J. Phys. Chem. 1981, 85, 3708-3712.

(34) Tessman, J. R.; Kahn, A. H.; Shockley, W. Electronic Polarizabilities of Ions in Crystals. Phys. Rev. 1953, 92, 890-895.

(35) Machmer, P. Empirical Correlation between the Molecular Electric Field Gradients and the Average Molecular Polarizabilities of Some Halogen Compounds. Nature 1968, 217, 165-166.

(36) Krishnamurti, K. A Theoretical Expression for Solubility Product. Nature 1958, 182, $1227-1228$. 
(37) Krishnamurti, K. Relation between the Polarizability of Ions and Other Significant Properties. Nature 1959, 183, 1043-1043.

(38) Volkov, A. G.; Paula, S.; Deamer, D. W. Two Mechanisms of Permeation of Small Neutral Nolecules and Hydrated Ions across Phospholipid Bilayers. Bioelectrochem. Bioenerg. 1997, 42, 153-160.

(39) Yu, H.; Whitfield, T. W.; Harder, E.; Lamoureux, G.; Vorobyov, I.; Anisimov, V. M.; MacKerell Jr, A. D.; Roux, B. Simulating Monovalent and Divalent Ions in Aqueous Solution Using a Drude Polarizable Force Field. J. Chem. Theory Comput. 2010, 6, 774786.

(40) Lamoureux, G.; Roux, B. Absolute Hydration Free Energy Scale for Alkali and Halide Ions Established from Simulations with a Polarizable Force Field. J. Phys. Chem. B 2006, 110, 3308-3322. 


\section{Graphical TOC Entry}

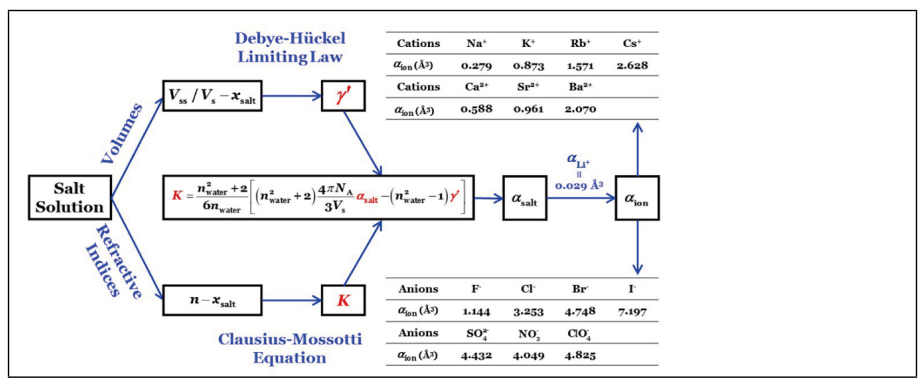

